\title{
$\mathrm{La}_{2} \mathrm{CoFeO}_{6}$ 竹节状中空纳米纤维的制备及其光催化性能
}

\author{
毕 军，吴艳波，赵恒彦，魏斌斌 \\ (大连交通大学 环境与化学工程学院, 大连 116028)
}

\begin{abstract}
摘 要: 采用静电纺丝法制得 $\mathrm{La}_{2} \mathrm{CoFeO}_{6}$ 竹节状中空纳米纤维光催化材料。 $\mathrm{La}_{2} \mathrm{CoFeO}_{6}$ 纳米纤维具有稳定的一维结 构, 由菱形晶型的 $\mathrm{La}_{2} \mathrm{CoFeO}_{6}$ 纳米颗粒相互连接组成, 并存在明显的竹节状中空结构, 其比表面积可达 $98.7 \mathrm{~m}^{2} / \mathrm{g}$ 。 $\mathrm{La}_{2} \mathrm{CoFeO}_{6}$ 纳米纤维对自然光具有较高的利用率, 其禁带宽度为 $1.6 \mathrm{eV}$ 。在甲基橙溶液浓度为 $10 \mathrm{mg} / \mathrm{L}, \mathrm{pH}$ 为 2 , 催 化剂用量为 $1.5 \mathrm{~g} / \mathrm{L}$ 条件下，自然光光照 $2 \mathrm{~h}$ 后, $\mathrm{La}_{2} \mathrm{CoFeO}_{6}$ 纳米纤维对甲基橙的降解率可达 $96.9 \%$ 。
\end{abstract}

关 键 词: $\mathrm{La}_{2} \mathrm{CoFeO}_{6}$ 纳米纤维; 静电纺丝; 光催化; 中空结构; 自然光

中图分类号: $\mathrm{O} 643$ 文献标识码: A

\section{Preparation and Photocatalytic Properties of $\mathrm{La}_{2} \mathrm{CoFeO}_{6}$ Bamboo-like Hollow Nanofibers}

\author{
BI Jun, WU Yan-Bo, ZHAO Heng-Yan, WEI Bin-Bin \\ (College of Environmental and Chemical Engineering, Dalian Jiaotong University, Dalian 116028, China)
}

\begin{abstract}
La}_{2} \mathrm{CoFeO}_{6}$ bamboo-like hollow nanofibers photocatalytic material was successfully prepared by electrostatic spinning. $\mathrm{La}_{2} \mathrm{CoFeO}_{6}$ nanofibers, which were connected to each other by rhombic $\mathrm{La}_{2} \mathrm{CoFeO}_{6}$ nanoparticles, had stable one-dimensional structure and clear bamboo-like hollow structure. The specific surface area of as-prepared inorganic nanofibers reached $98.7 \mathrm{~m}^{2} / \mathrm{g}$. $\mathrm{La}_{2} \mathrm{CoFeO}_{6}$ nanofibers had a high utilization for natural light and its band gap was $1.6 \mathrm{eV}$. When concentration of methyl orange was $10 \mathrm{mg} / \mathrm{L}$ with $\mathrm{pH}$ of 2 and the dosage of $\mathrm{La}_{2} \mathrm{CoFeO}_{6}$ nanofibers was $1.5 \mathrm{~g} / \mathrm{L}$, methyl orange was degraded by $\mathrm{La}_{2} \mathrm{CoFeO}_{6}$ nanofibers within $2 \mathrm{~h}$ under natural light at $96.9 \%$.
\end{abstract}

Key words: $\mathrm{La}_{2} \mathrm{CoFeO}_{6}$ nanofibers; electrostatic spinning; photocatalysis; hollow structure; natural light

半导体光催化剂可以直接利用太阳能降解有 机污染物, 在环境和能源领域具有广泛的应用前 景。现阶段使用较普遍的是结构稳定且无毒的 $\mathrm{TiO}_{2}{ }^{[1]}$, 但是 $\mathrm{TiO}_{2}$ 的禁带宽度大, 难以回收、分离 制约了它的发展和应用。

钙钛矿型金属氧化物是继 $\mathrm{TiO}_{2}$ 之后被提出的 另一类新型光催化剂, 以其具有独特的晶体结构 ${ }^{[2] 、}$ 灵活的 “化学剪裁性” ${ }^{[3]}$ 以及较好的光催化活性(光 响应范围延伸至可见光区), 受到广泛关注 ${ }^{[4]}$ 。双钙 钛矿型金属氧化物 $\left(\mathrm{A}_{2} \mathrm{~B}^{1} \mathrm{~B}^{2} \mathrm{O}_{6}\right)$ 是一种结构特殊的钻
钛矿型金属氧化物 ${ }^{[5]}$, 在其分子结构中, $\mathrm{B}$ 位原子的 八面体结构由 $\mathrm{B}^{1} \mathrm{O}_{6}$ 和 $\mathrm{B}^{2} \mathrm{O}_{6}$ 有序排列而成, 形成了 连续的 $\mathrm{B}^{1}-\mathrm{O}-\mathrm{B}^{2}$ 结构, 与 $\mathrm{ABO}_{3}$ 型钙钛矿的分子结 构相比, 这种有序结构更有利于电荷在原子间的传 递 ${ }^{[6]}$, 因此, 双钙钛矿型金属氧化物表现出更加良 好的半导体及光/电性能 ${ }^{[7]}$ 。Iwakura 等 ${ }^{[8]}$ 对双钙钛矿 中金属阳离子的排列与材料光催化性能的关系进行 了研究; $\mathrm{Li}$ 等 ${ }^{[9]}$ 利用固相合成法制备出 $\mathrm{Ca}_{2} \mathrm{NiWO}_{6}$ 光催化材料, 并对该材料在不同光照条件下催化分 解水的活性进行了研究。目前, 双钻钛矿纳米材料 
的制备大多局限于传统制备方法，且产物通常为 粉末状 ${ }^{[10]}$ 。但是由于粉末状催化剂团聚现象严重, 使得双钻钛矿型光催化材料的催化性能受到了严 重影响。

近年来, 随着科学技术的发展, 钙钛矿型纳米 纤维因其具有比表面积大、长径比大、不易团聚等 优点 ${ }^{[11]}$, 在光催化领域受到了广泛关注。一般通过 AAO 模板法、静电纺丝法均可制得钙钛矿型一维纳 米材料, 其中, 静电纺丝法设备简单、无污染, 是制 备高长径比钙钛矿型纳米纤维的理想方法。Wang 等 ${ }^{[12]}$ 利用静电纺丝技术制备了 $\mathrm{Ba} 、 \mathrm{Mn}$ 掺杂 $\mathrm{BiFeO}_{3}$ 纳米纤维光催化材料; Ren 等 ${ }^{[13]}$ 采用电纺技术结合水 热法得到了 $\mathrm{ZnO} / \mathrm{BaTiO}_{3}$ 纳米纤维异质结构, 并对其 在紫外光下的光催化活性进行了研究; Dong 等 ${ }^{[14]}$ 利 用静电纺丝法制得 $\mathrm{LaCoO}_{3}$ 纳米纤维, 并对该材料 的最佳降解条件进行了探索。但是, 静电纺丝法 制备的钲钛矿主要集中于 $\mathrm{ABO}_{3}$ 型, 对 $\mathrm{A}_{2} \mathrm{~B}^{1} \mathrm{~B}^{2} \mathrm{O}_{6}$ 型纳米纤维的研究却很少。本工作采用静电纺丝 技术, 经预氧化和炦烧制得竹节状中空结构的双 钙钛矿型 $\mathrm{La}_{2} \mathrm{CoFeO}_{6}$ 纳米纤维，并以甲基橙作为 降解物, 在自然光下考察 $\mathrm{La}_{2} \mathrm{CoFeO}_{6}$ 纳米纤维的 光催化性能。

\section{1 实验方法}

\section{1 药品}

聚乙烯吡咯烷酮(PVP, K90, $M_{\mathrm{w}}=1300000$, 天津 市博迪化工有限公司); N, N-二甲基甲酰胺(DMF, 天津市富宇精细化工有限公司, 分析纯); 硝酸镧 $\left(\mathrm{La}\left(\mathrm{NO}_{3}\right)_{3} \cdot n \mathrm{H}_{2} \mathrm{O}\right.$, 国药集团化学试剂有限公司, 分 析纯); 九水硝酸铁 $\left(\mathrm{Fe}\left(\mathrm{NO}_{3}\right)_{3} \cdot 9 \mathrm{H}_{2} \mathrm{O}\right.$, 西陇化工股份 有限公司, 分析纯); 乙酸钴 $\left(\mathrm{Co}\left(\mathrm{CH}_{3} \mathrm{COO}\right)_{2} \cdot 4 \mathrm{H}_{2} \mathrm{O}\right.$, 天津市光复精细化工研究所, 分析纯); 甲基橙(国 药集团化学试剂有限公司)。

\section{$1.2 \mathrm{La}_{2} \mathrm{CoFeO}_{6}$ 纳米纤维的制备}

将 $3.100 \mathrm{~g} \mathrm{PVP}$ 加入到 $20 \mathrm{~mL}$ DMF 中, 搅拌溶 解。分别称取 $1.300 \mathrm{~g} \mathrm{La}\left(\mathrm{NO}_{3}\right)_{3} \cdot n \mathrm{H}_{2} \mathrm{O} 、 0.498 \mathrm{~g}$ $\mathrm{Co}\left(\mathrm{CH}_{3} \mathrm{COO}\right)_{2} \cdot 4 \mathrm{H}_{2} \mathrm{O}$ 和 $0.808 \mathrm{~g} \mathrm{Fe}\left(\mathrm{NO}_{3}\right)_{3} \cdot 9 \mathrm{H}_{2} \mathrm{O}$ 加入 到上述溶液中, 搅拌均匀, 配制成纺丝前驱体溶液。 采用静电纺丝法, 控制接收距离为 $15 \mathrm{~cm}$, 纺丝电 压为 $15 \mathrm{kV}$, 制得聚乙烯吡咯烷酮/硝酸镧-乙酸钴硝酸铁 (PVP/LCF)复合纳米纤维。将复合纳米纤维 置于管式炉内, 在 $220^{\circ} \mathrm{C}$ 空气中预氧化 $1 \mathrm{~h}$, 升温速 率为 $1^{\circ} \mathrm{C} / \mathrm{min}$; 然后, 在 $700^{\circ} \mathrm{C}$ 空气气氛中炦烧 $3 \mathrm{~h}$, 升温速率为 $2^{\circ} \mathrm{C} / \mathrm{min}$, 制得 $\mathrm{La}_{2} \mathrm{CoFeO}_{6}$ 竹节状中空纳
米纤维。

\section{3 表征}

利用 $\mathrm{X}$ 射线衍射 (XRD, XRD-6000, 日本 Shimadzu 公司)、场发射扫描电镜(FE-SEM, SUPRA 55, 德国 Carl Zeiss Jena 公司)、傅里叶变换红外光 谱(FT-IR, Nicolet 8700, 美国 Thermo Electron 公 司)、透射电镜(TEM，JEM-2100F，日本 JEOL 公司) 和比表面积分析仪 (BET, ASAP 2020, 美国 Micromeritics 公司)对 $\mathrm{La}_{2} \mathrm{CoFeO}_{6}$ 竹节状中空纳米纤 维的形貌及物相结构进行表征; 利用紫外可见光谱 仪(UV-Vis，UV-3802S，尤尼柯上海仪器有限公司) 对纳米纤维光学性质进行检测。

\section{4 可见光催化测试}

在自然光下，以浓度为 $10 \mathrm{mg} / \mathrm{L}$ 的甲基橙作为 降解物, 将一定质量的 $\mathrm{La}_{2} \mathrm{CoFeO}_{6}$ 竹节状中空纳米 纤维加入到甲基橙溶液中, 进行光催化性能测试。 每隔相同时间利用紫外-可见分光光度计(UV752N, 上海佑科仪器仪表有限公司)在 $463 \mathrm{~nm}$ 测定吸光度 值，并根据公式(1)计算降解率。

$$
\eta=\left[\left(A_{0}-A\right) / A_{0}\right] \times 100 \%
$$

式中, $A_{0}$ 为甲基橙溶液的初始吸光度值, $A$ 为降解后 溶液的吸光度值, $\eta$ 为降解率。

\section{2 结果与讨论}

\subsection{XRD 分析}

图 1 为 $\mathrm{La}_{2} \mathrm{CoFeO}_{6}$ 纳米纤维的 XRD 图谱, 主要 的衍射峰出现在 $2 \theta=23.0^{\circ} 、 32.6^{\circ} 、 32.9^{\circ} 、 38.6^{\circ} 、 40.3^{\circ}$ 、 $47.0^{\circ} 、 52.7^{\circ} 、 53.2^{\circ} 、 58.3^{\circ} 、 68.2^{\circ} 、 69.1^{\circ} 、 77.8^{\circ}$ 和 $78.5^{\circ}$ 的位置, 与菱形晶型 $\mathrm{La}_{2} \mathrm{CoFeO}_{6}$ 标准卡片 (PDF-00-053-1211)中的衍射峰相吻合, 且上述特征 峰分别与菱形晶型 $\mathrm{La}_{2} \mathrm{CoFeO}_{6}$ 的(012)、(110)、(104)、

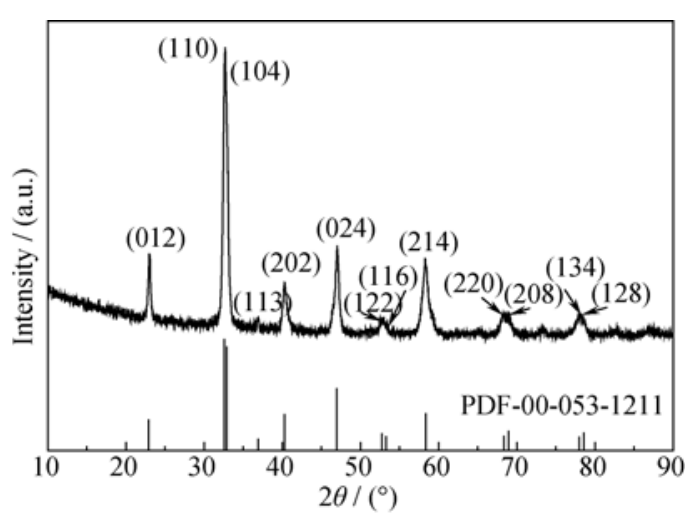

图 $1 \mathrm{La}_{2} \mathrm{CoFeO}_{6}$ 纳米纤维的 $\mathrm{XRD}$ 图谱

Fig. 1 XRD pattern of $\mathrm{La}_{2} \mathrm{CoFeO}_{6}$ nanofibers 
(113)、(202)、(024)、(122)、(116)、(214)、(220)、 (208)、(134)、(128)晶面相对应, 表明纤维主体由菱 形晶型的 $\mathrm{La}_{2} \mathrm{CoFeO}_{6}$ 组成, 晶体结构相对完整且纯 度较高。

\subsection{FE-SEM 分析}

图 2 为 $\mathrm{La}_{2} \mathrm{CoFeO}_{6}$ 纳米纤维的 FE-SEM 照片, 可以看出, $\mathrm{La}_{2} \mathrm{CoFeO}_{6}$ 纳米纤维保持着稳定的一维 结构及较高的长径比, 纤维由 $\mathrm{La}_{2} \mathrm{CoFeO}_{6}$ 颗粒相互 连接组装而成, 颗粒粒径均一, 并且纤维间没有因 高温而发生交联或熔并现象, 呈空间网状结构。

\subsection{FT-IR 分析}

图 3 为 $\mathrm{La}_{2} \mathrm{CoFeO}_{6}$ 纳米纤维 FT-IR 谱图, 由图 可知, PVP/LCF 复合纳米纤维经 $700^{\circ} \mathrm{C}$ 㷽烧后纤维 中有机物的吸收谱带基本消失, 在 1000 和 $2900 \mathrm{~cm}^{-1}$ 附近出现的弱吸收峰为炦烧后纤维中残余的极少量 的 $-\mathrm{C}-\mathrm{C}-$ 键及样品表面微量的 $-\mathrm{C}-\mathrm{H}$ 键的振动峰; 在 597 和 $402 \mathrm{~cm}^{-1}$ 附近出现的吸收峰为炦烧后形成的
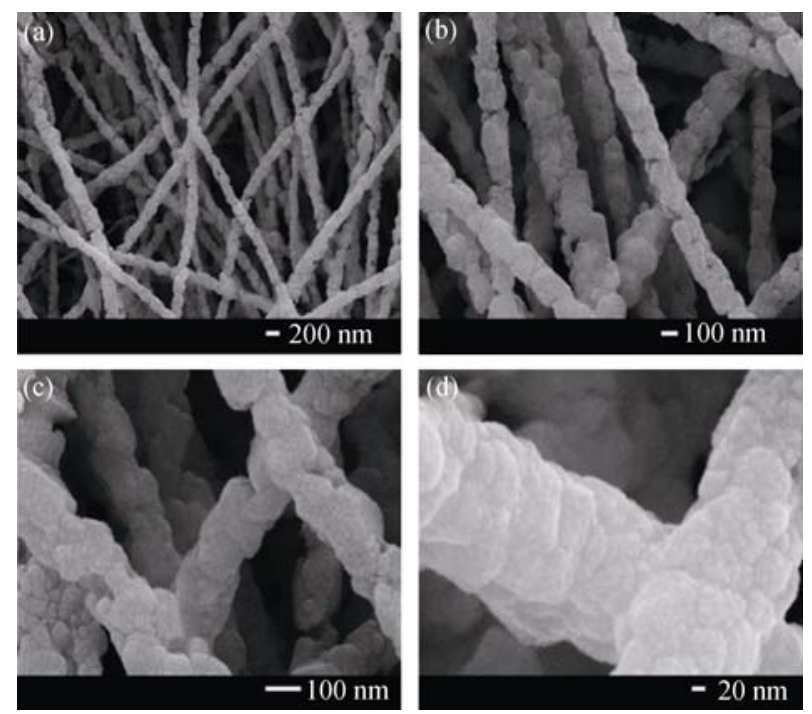

图 2 不同放大倍数下的 $\mathrm{La}_{2} \mathrm{CoFeO}_{6}$ 纳米纤维的 FE-SEM 图片 Fig. 2 FE-SEM images of $\mathrm{La}_{2} \mathrm{CoFeO}_{6}$ nanofibers at different magnification

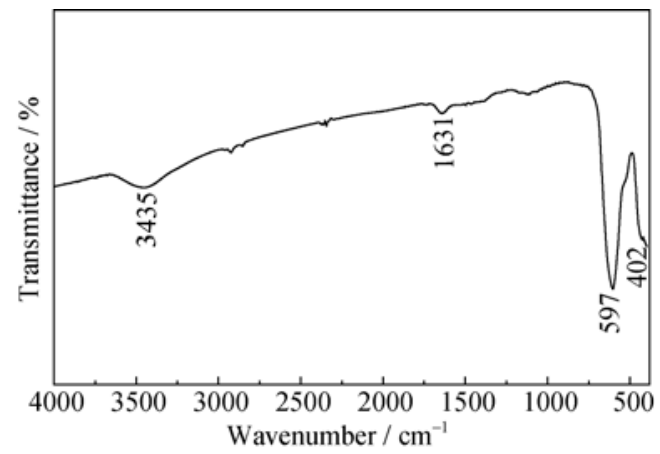

图 $3 \mathrm{La}_{2} \mathrm{CoFeO}_{6}$ 纳米纤维 FT-IR 谱图

Fig. 3 FT-IR spectrum of $\mathrm{La}_{2} \mathrm{CoFeO}_{6}$ nanofibers
金属氧键的振动峰 ${ }^{[15-16]}$ 。此外, 在 3435 及 $1631 \mathrm{~cm}^{-1}$ 处出现的吸收峰分别归因于材料表面的 $-\mathrm{OH}$ 及吸 附水分子中 $\mathrm{H}-\mathrm{O}$ 键的伸缩振动，而材料表面存在的 $-\mathrm{OH}$ 有利于增加催化过程中氢氧自由基的生成数 量, 提高了材料光催化活性 ${ }^{[17]}$ 。

\subsection{TEM 分析}

对 $\mathrm{La}_{2} \mathrm{CoFeO}_{6}$ 纳米纤维的微结构进行 TEM 测 试, 如图 4 所示。由图 4(a,b)可以看出, 纤维的直径 介于 $120 ~ 250 \mathrm{~nm} ; \mathrm{La}_{2} \mathrm{CoFeO}_{6}$ 纳米颗粒的平均粒径 为 $55 \mathrm{~nm}$; 纤维具有明显的竹节状中空结构, 在纤 维内部存在连续排列的椭圆形空腔, 空腔直径在 $100 \mathrm{~nm}$ 左右。这是由于煅烧时, 聚集在高聚物纤维 表面的金属盐快速分解，生成的 $\mathrm{La}_{2} \mathrm{CoFeO}_{6}$ 纳米颗 粒相互结合形成具有稳定结构的金属氧化物外壳, 同时, PVP 受热分解，在纤维内部形成空腔结构，最 终形成了中空结构 ${ }^{[18]}$ 。从 $\mathrm{La}_{2} \mathrm{CoFeO}_{6}$ 纳米纤维的选 区电子衍射图片(图 4(c)) 可以看出, $\mathrm{La}_{2} \mathrm{CoFeO}_{6}$ 晶体 颗粒的衍射斑点呈环状排列, 可以判断 $\mathrm{La}_{2} \mathrm{CoFeO}_{6}$ 竹 节状中空纳米纤维为单相多晶结构, 由 $\mathrm{La}_{2} \mathrm{CoFeO}_{6}$ 颗 粒相互连接组装而成, 这与 FE-SEM 分析结果相符 合。由高分辨透射电镜分析(图 4(d)) 可知, 晶面间距 为 $0.267 \mathrm{~nm}$, 与菱形双钙钛矿 $\mathrm{La}_{2} \mathrm{CoFeO}_{6}$ 晶体结构 中(104)晶面的晶面间距一致, 从而进一步证明, 竹节 状中空纳米纤维由 $\mathrm{La}_{2} \mathrm{CoFeO}_{6}$ 纳米颗粒构成。

\subsection{BET 分析}

图 5 为 $\mathrm{La}_{2} \mathrm{CoFeO}_{6}$ 竹节状中空纳米纤维的 $\mathrm{N}_{2}$ 吸脱附等温线, 可以看出, 纤维的吸脱附等温线属 于典型的 IV 型等温线, 吸附体积主要是 $\mathrm{La}_{2} \mathrm{CoFeO}_{6}$ 纳米颗粒的堆积间隙造成的; 相对压力 $\left(p / p_{0}\right)$ 在 $0.4 \sim 1.0$ 范围内可观察到明显的 H1 型滞后环, 这是
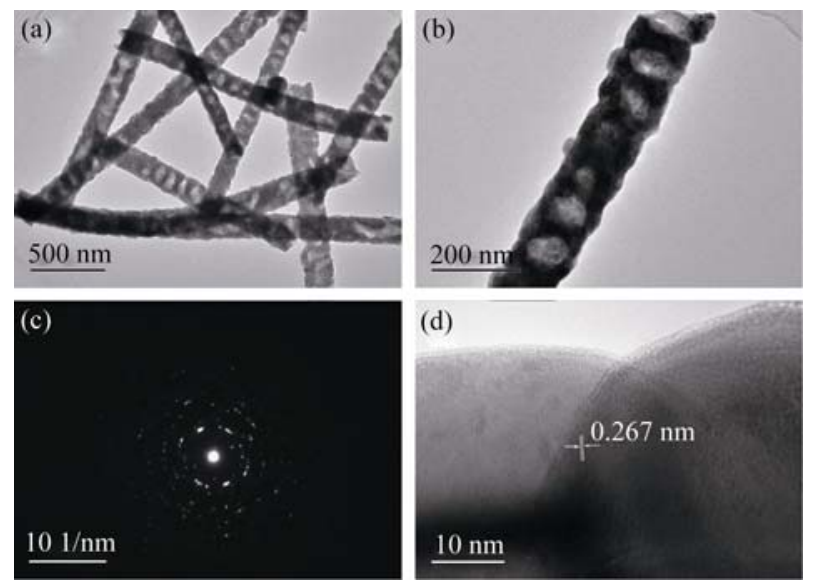

图 $4 \mathrm{La}_{2} \mathrm{CoFeO}_{6}$ 纳米纤维的 TEM 照片 $(\mathrm{a}, \mathrm{b})$ 、选区电子衍 射图案(c)和高分辨率透射电镜照片 (d)

Fig. 4 TEM images (a, b), SEAD pattern (c) and HRTEM image (d) of $\mathrm{La}_{2} \mathrm{CoFeO}_{6}$ nanofibers 


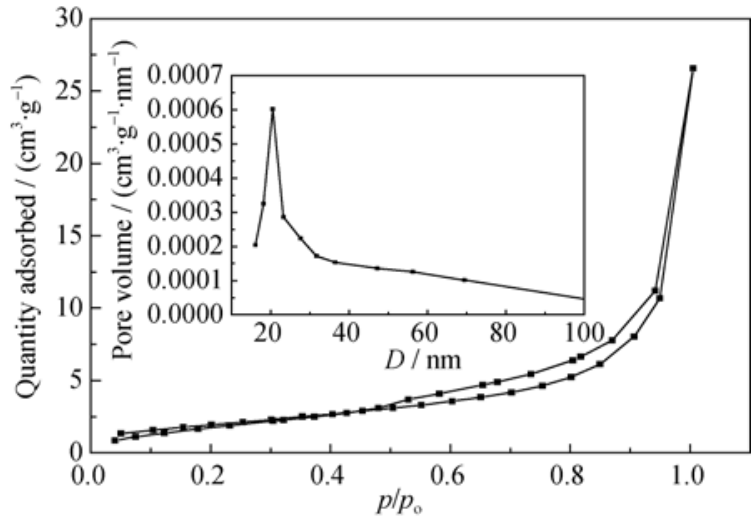

图 $5 \mathrm{La}_{2} \mathrm{CoFeO}_{6}$ 竹节状中空纳米纤维的 $\mathrm{N}_{2}$ 吸脱附等温线和 孔径分布曲线(插图)

Fig. $5 \mathrm{~N}_{2}$ adsorption-desorption isotherms and pore size distribution curves (inset) of $\mathrm{La}_{2} \mathrm{CoFeO}_{6}$ bamboo-like hollow nanofibers

由于纤维中存在介孔 $(2 \sim 50 \mathrm{~nm})$ 结构造成的; 同时, 在 $p / p_{0}$ 接近 1.0 时, $\mathrm{N}_{2}$ 吸附量急剧增加, 表明纤维中 $\mathrm{La}_{2} \mathrm{CoFeO}_{6}$ 纳米颗粒之间存在很大空隙, 这有利于 光催化过程中反应物的吸附及产物的扩散。由 $\mathrm{La}_{2} \mathrm{CoFeO}_{6}$ 竹节状中空纳米纤维的孔径分布曲线(图 5 插图)可知, $\mathrm{La}_{2} \mathrm{CoFeO}_{6}$ 纳米纤维主要以介孔结构 为主, 纤维中还存在一定量的大孔(大于 $50 \mathrm{~nm}$ ) 结 构, 丰富的孔径分布使得 $\mathrm{La}_{2} \mathrm{CoFeO}_{6}$ 纳米纤维具有 较高的比表面积, 其比表面积可达 $98.7 \mathrm{~m}^{2} / \mathrm{g}$ 。

\section{$2.6 \mathrm{UV}-\mathrm{Vis}$ 分析}

图 6 为 $\mathrm{La}_{2} \mathrm{CoFeO}_{6}$ 竹节状中空纳米纤维的 UV-Vis 谱图, 由图可知, $\mathrm{La}_{2} \mathrm{CoFeO}_{6}$ 竹节状中空纳 米纤维的吸收光谱在 300 800 $\mathrm{nm}$ 波段范围内具有 较高的吸收强度, 这有利于催化剂更好地利用自然 光。 $\mathrm{La}_{2} \mathrm{CoFeO}_{6}$ 竹节状中空纳米纤维的禁带宽度可 根据公式(2) ${ }^{[19]}$ 计算。

$$
\alpha \mathrm{h} v=\mathrm{A}(\mathrm{h} v-E \mathrm{~g})^{n}
$$

式中, $\alpha$ 为吸收系数, $h v$ 为光能量, $\mathrm{A}$ 为常数, $E_{\mathrm{g}}$ 为禁

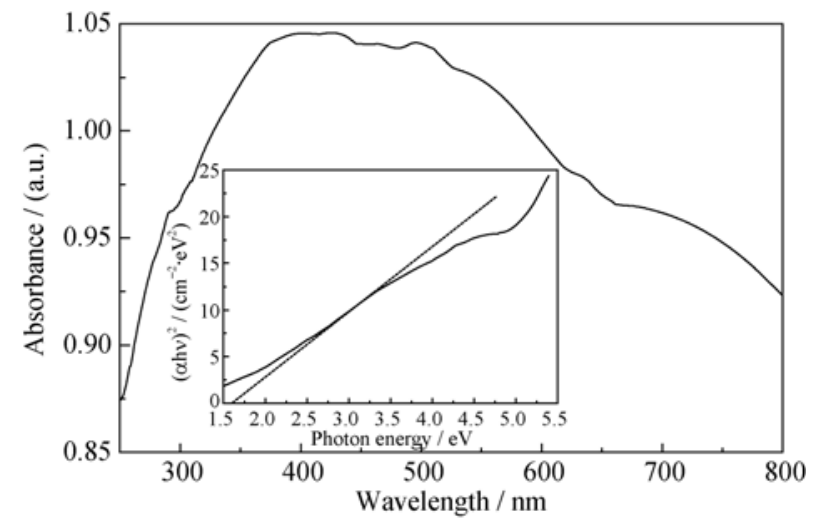

图 $6 \mathrm{La}_{2} \mathrm{CoFeO}_{6}$ 竹节状中空纳米纤维的 UV-Vis 谱图

Fig. $6 \mathrm{UV}-\mathrm{Vis}$ spectra of $\mathrm{La}_{2} \mathrm{CoFeO}_{6}$ bamboo-like hollow nanofibers
带宽度，间接跃迁时 $n$ 取 2, 直接跃迁时 $n$ 取 0.5。

根据文献报道, $\mathrm{La}_{2} \mathrm{CoFeO}_{6}$ 为直接跃迁型半导 体 ${ }^{[20-22]}$ 。因此, $\mathrm{La}_{2} \mathrm{CoFeO}_{6}$ 纳米纤维的禁带宽度可通 过做 $(\alpha \mathrm{h} v)^{2} \sim \mathrm{h} v$ 曲线(图 6 插图)并作曲线在近吸收边 位置的切线获得, $\mathrm{La}_{2} \mathrm{CoFeO}_{6}$ 纳米纤维的禁带宽度 约为 $1.6 \mathrm{eV}$, 表明 $\mathrm{La}_{2} \mathrm{CoFeO}_{6}$ 纳米纤维具有较窄的 禁带宽度，电子从价带跃迁到导带时所需的能量更 小, 对自然光的利用率较高, 是一种具有发展潜力 的新型可见光光催化材料。

\section{7 光催化活性测试}

选取甲基橙溶液浓度为 $10 \mathrm{mg} / \mathrm{L}$, 考察 $\mathrm{La}_{2} \mathrm{CoFeO}_{6}$ 竹节状中空纳米纤维的光催化性能。由图 7 可见, 在 无光照、加入 $1 \mathrm{~g} / \mathrm{L}$ 的 $\mathrm{La}_{2} \mathrm{CoFeO}_{6}$ 纳米纤维的条件 下, 静置 $2 \mathrm{~h}$ 后, 甲基橙的降解量仅为 $5.3 \%$, 这是由 纤维对甲基橙的吸附作用造成的，表明在无光照条 件下 $\mathrm{La}_{2} \mathrm{CoFeO}_{6}$ 纳米纤维对甲基橙无催化活性。在 有光照、无 $\mathrm{La}_{2} \mathrm{CoFeO}_{6}$ 纳米纤维的条件下, 自然光 光照 $2 \mathrm{~h}$ 后，甲基橙几乎不发生分解，表明甲基橙在 光照时间内具有很好的稳定性, 其自敏化降解反应 的影响可以忽略; 在 $\mathrm{La}_{2} \mathrm{CoFeO}_{6}$ 纳米纤维的加入量 为 $1 \mathrm{~g} / \mathrm{L}$ 的条件下, 自然光光照 $2 \mathrm{~h}$ 后, 甲基橙的降 解率为 $74.2 \%$; 而在相同条件下采用 $\mathrm{TiO}_{2}(\mathrm{P} 25)$ 作为 光催化材料时, 只有 $21 \%$ 的甲基橙被降解, 表明在 自然光光照条件下, $\mathrm{La}_{2} \mathrm{CoFeO}_{6}$ 纳米纤维对甲基橙 具有更高的光催化活性。

选定甲基橙溶液 $\mathrm{pH}$ 为中性, 考察 $\mathrm{La}_{2} \mathrm{CoFeO}_{6}$ 纳米纤维的加入量对降解率的影响, 如图 8(a)所示。 当催化剂的加入量小于 $1.5 \mathrm{~g} / \mathrm{L}$ 时, 甲基橙溶液的降 解率随着 $\mathrm{La}_{2} \mathrm{CoFeO}_{6}$ 纳米纤维加入量的增加而增加, 这是由于增加 $\mathrm{La}_{2} \mathrm{CoFeO}_{6}$ 纳米纤维的加入量, 使溶 液中参与光催化反应的活性位增多, 在光降解过程 中产生了足够多的氢氧自由基 $(\cdot \mathrm{OH})$ 加快了光催化 降解速率; 此外, 由于 $\mathrm{La}_{2} \mathrm{CoFeO}_{6}$ 纳米纤维具有独

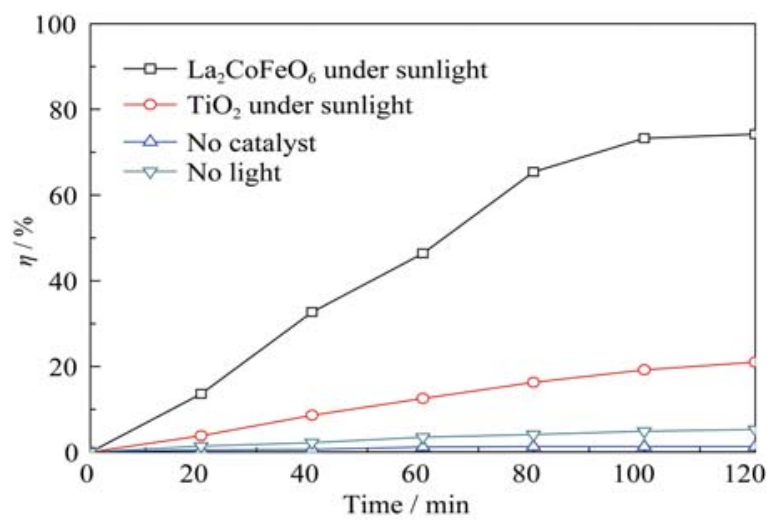

图 7 不同条件下甲基橙溶液 $(\mathrm{pH}=5 \sim 6)$ 的降解率变化曲线

Fig. 7 Degradation rate curves of methyl orange solution $(\mathrm{pH}=5-6)$ under different conditions 

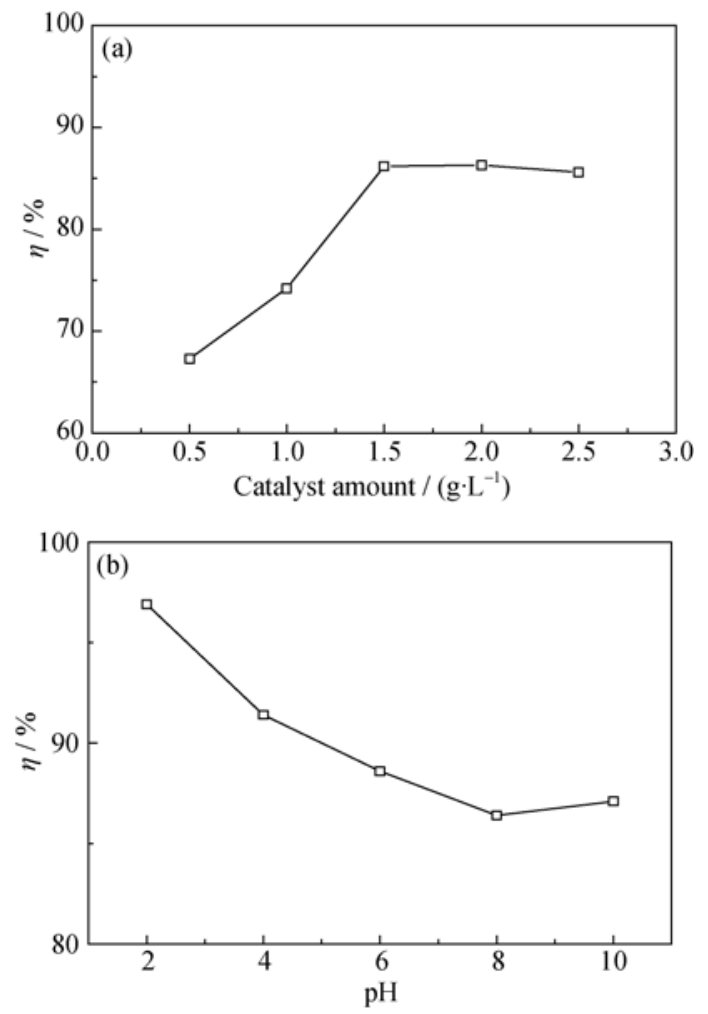

图 8 催化剂加入量(a)和溶液 $\mathrm{pH}(\mathrm{b})$ 对 $\mathrm{La}_{2} \mathrm{CoFeO}_{6}$ 竹节状中 空纳米纤维光催化性能的影响

Fig. 8 Effect of catalyst amount (a) and solution $\mathrm{pH}(\mathrm{b})$ on the photocatalytic properties of $\mathrm{La}_{2} \mathrm{CoFeO}_{6}$ bamboo-like hollow nanofibers

特的竹节状中空结构, 材料的比表面积较大, 为光催 化反应提供了更多的反应空间, 并且在 $\mathrm{La}_{2} \mathrm{CoFeO}_{6}$ 分 子结构中 $\mathrm{B}$ 位原子的八面体结构由 $\mathrm{CoO}_{6}$ 和 $\mathrm{FeO}_{6}$ 有 序排列而成, 形成连续的 $\mathrm{Co}-\mathrm{O}-\mathrm{Fe}$ 结构, 为电荷的快 速迁移提供了通道 ${ }^{[6]}$, 促进了光催化反应的进行。但 是, 当催化剂的加入量大于 $1.5 \mathrm{~g} / \mathrm{L}$ 时, 甲基橙溶液 的降解率维持在 $87.4 \%$ 不再发生明显变化, 这是由 于 $\mathrm{La}_{2} \mathrm{CoFeO}_{6}$ 纳米纤维在甲基橙溶液中处于悬浮状 态, 若加入量过多, 会使溶液浊度增加, 对入射光 产生屏蔽散射效应 ${ }^{[23]}$, 影响催化剂的降解效率。因 此, $\mathrm{La}_{2} \mathrm{CoFeO}_{6}$ 纳米纤维的最佳加入量为 $1.5 \mathrm{~g} / \mathrm{L}$ 。

选定 $\mathrm{La}_{2} \mathrm{CoFeO}_{6}$ 纳米纤维的加入量为 $1.5 \mathrm{~g} / \mathrm{L}$, 考察甲基橙溶液 $\mathrm{pH}$ 对降解率的影响, 如图 8(b)所 示。从图 8(b) 可以看出, 在碱性条件下, $\mathrm{La}_{2} \mathrm{CoFeO}_{6}$ 纳米纤维的光催化性能几乎不发生变化; 而在酸性 条件下, $\mathrm{La}_{2} \mathrm{CoFeO}_{6}$ 纳米纤维的催化活性随 $\mathrm{pH}$ 的减 小而增大, 当溶液的 $\mathrm{pH}$ 为 2 时, 催化剂对甲基橙溶 液的降解率可达 $96.9 \%$, 表明 $\mathrm{La}_{2} \mathrm{CoFeO}_{6}$ 纳米纤维 在酸性环境中具有更高的光催化活性。

在自然光光照 $2 \mathrm{~h}$, 甲基橙溶液的 $\mathrm{pH}$ 为 2 , $\mathrm{La}_{2} \mathrm{CoFeO}_{6}$ 纳米纤维加入量为 $1.5 \mathrm{~g} / \mathrm{L}$ 的条件下, 对
$\mathrm{La}_{2} \mathrm{CoFeO}_{6}$ 竹节状中空纳米纤维进行光降解循环测 试, 如图 9 所示。从图 9 可以看出, 纤维的光催化活 性在每次循环后均有所下降, 这是由于酸性条件下 $\mathrm{La}_{2} \mathrm{CoFeO}_{6}$ 被缓慢消耗所致。但是, 经过 5 次循环后, 仍有 79.6\%的甲基橙被降解，表明 $\mathrm{La}_{2} \mathrm{CoFeO}_{6}$ 纳米 纤维是一种具有良好稳定性的可见光光催化材料。

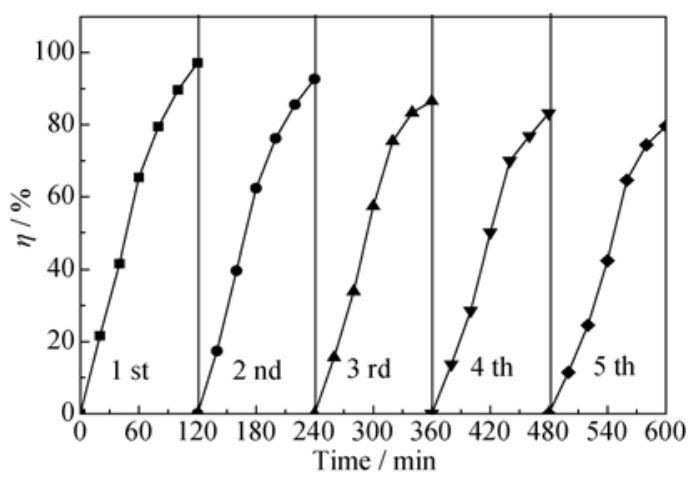

图 $9 \mathrm{La}_{2} \mathrm{CoFeO}_{6}$ 竹节状中空纳米纤维的光催化稳定性

Fig. 9 Photocatalytic stability of $\mathrm{La}_{2} \mathrm{CoFeO}_{6}$ bamboo-like hollow nanofibers

\section{3 结论}

采用静电纺丝法制得 $\mathrm{La}_{2} \mathrm{CoFeO}_{6}$ 竹节状中空纳 米纤维。纤维的直径介于 $120 \sim 250 \mathrm{~nm}$, 比表面积为 $98.7 \mathrm{~m}^{2} / \mathrm{g}$, 由菱形晶型的 $\mathrm{La}_{2} \mathrm{CoFeO}_{6}$ 纳米颗粒相互 连接组装而成, 颗粒的平均粒径为 $55 \mathrm{~nm}$ 。纤维内部 具有连续排列的椭圆形空腔, 空腔的平均直径为 $100 \mathrm{~nm}$, 此结构为光催化过程中反应物的吸附及生 成物的扩散提供了更多的空间，从而有效提高了其 催化性能。并且, $\mathrm{La}_{2} \mathrm{CoFeO}_{6}$ 纳米纤维光催化剂的禁 带宽度仅为 $1.6 \mathrm{eV}$, 电子从价带跃迁到导带时所需 的能量更小, 使得 $\mathrm{La}_{2} \mathrm{CoFeO}_{6}$ 纳米纤维对自然光具 有较高的利用率。

\section{参考文献:}

[1] QU XIAO-FEI, LIU LU-YING, LI XUE-QIN, et al. Preparation of $\mathrm{TiO}_{2}$ : $\mathrm{E}^{3+}$ hollow spheres and its photocatalytic properties. Journal of Inorganic Materials, 2015, 30(2): 183-188.

[2] INOUE S, KAWAI M, ICHIKAWA N, et al. Anisotropic oxygen diffusion at low temperature in perovskite- structure iron oxides. Nature Chemistry, 2010, 2(3): 213-217.

[3] TIEN-THAO N, ALAMDARI H, ZAHEDI-NIAKI M H, et al. $\mathrm{LaCo}_{1-x} \mathrm{Cu}_{x} \mathrm{O}_{3-\delta}$ perovskite catalysts for higher alcohol synthesis. Applied Catalysis A: General, 2006, 311: 204-212.

[4] LI YUE-JUN, CAO TIE-PING, MEI ZE-MIN. Preparation and photocatalytic properties of $\mathrm{BaTiO}_{3} / \mathrm{TiO}_{2}$ heterostructured nanofi 
bers. Journal of Inorganic Materials, 2014, 29(7): 741-746.

[5] DU C H, ADUR R, WANG H L, et al. Control of magnetocrystalline anisotropy by epitaxial strain in double perovskite $\mathrm{Sr}_{2} \mathrm{FeMoO}_{6}$ films. Physical Review Letters, 2013, 110(14-5): 147204.

[6] KIM G, WANG S, JACOBSON A J, et al. Rapid oxygen ion diffusion and surface exchange kinetics in $\mathrm{PrBaCo}_{2} \mathrm{O}_{5+x}$ with a perovskite related structure and ordered A cations. Journal of Materials Chemistry, 2007, 17(24): 2500-2505.

[7] WU YAN-BO, BI JUN, WEI BIN-BIN. Preparation and photocatalytic properties of $\mathrm{La}_{2} \mathrm{CoFeO}_{6}$ bamboo-like hollow nanofibers. Acta Physico-Chimica Sinica, 2015, 31(2): 315-321.

[8] IWAKURA H, EINAGA H, TERAOKA Y. Relationship between cation arrangement and photocatalytic activity for $\mathrm{Sr}-\mathrm{Al}-\mathrm{Nb}-\mathrm{O}$ double perovskite. Inorganic Chemistry, 2010, 49(24): 1136211369.

[9] LI D F, ZHENG J, ZOU Z G. Band structure and photocatalytic properties of perovskite-type compound $\mathrm{Ca}_{2} \mathrm{NiWO}_{6}$ for water splitting. Journal of Physics and Chemistry of Solids, 2006, 67(4): 801- 806 .

[10] HUANG Y F, LI Y B, WEI Y L, et al. Photocatalytic property of partially substituted Pt-intercalated layered perovskite, $\mathrm{ASr}_{2} \mathrm{Ta}_{x} \mathrm{Nb}_{3-\mathrm{x}} \mathrm{O}_{10}$ $(\mathrm{A}=\mathrm{K}, \mathrm{H} ; x=0,1,1.5,2$ and 3). Solar Energy Materials and Solar Cells, 2011, 95(3): 1019-1027.

[11] KODAMBAKA S, TERSOFF J, REUTER M C, et al. Germanium nanowire growth below the eutectic temperature. Science, 2007, 316(5825): 729-732.

[12] WANG H C, LIN Y H, FENG Y N, et al. Photocatalytic behaviors observed in $\mathrm{Ba}$ and $\mathrm{Mn}$ doped $\mathrm{BiFeO}_{3}$ nanofibers. Journal of Electroceramics, 2013, 31(1/2): 271-274.

[13] REN P R, FAN H Q, WANG X. Electrospun nanofibers of $\mathrm{ZnO} / \mathrm{BaTiO}_{3}$ heterostructures with enhanced photocatalytic activity. Catalysis Communications, 2012, 25: 32-35.

[14] DONG B, LI Z C, LI Z Y, et al. Highly efficient $\mathrm{LaCoO}_{3}$ nanofibers catalysts for photocatalytic degradation of Rhodamine $\mathrm{B}$.
Journal of the American Ceramic Society, 2010, 93(11): $3587-$ 3590 .

[15] LI S D, JING L Q, FU W, et al. Photoinduced charge property of nanosized perovskite-type $\mathrm{LaFeO}_{3}$ and its relationships with photocatalytic activity under visible irradiation. Materials Research Bulletin, 2007, 42(2): 203-212.

[16] JIA L S, LI J J, FANG W P. Enhanced visible-light active C and Fe co-doped $\mathrm{LaCoO}_{3}$ for reduction of carbon dioxide. Catalysis Communications, 2009, 11(2): 87-90.

[17] SHEN Y, ZHAO Q D, LI X Y, et al. Monodisperse $\mathrm{Ca}_{0.15} \mathrm{Fe}_{2.85} \mathrm{O}_{4}$ microspheres: facile preparation, characterization, and optical properties. Journal of Materials Science, 2012, 47(7): 3320-3326.

[18] 王进贤, 董相廷. 静电纺丝技术与无机纳米材料合成. 北京: 国防工业出版社, 2012: 136-137.

[19] ZHAO W R, WANG Y, YANG Y, et al. Carbon spheres supported visible-light-driven $\mathrm{CuO}-\mathrm{BiVO}_{4}$ heterojunction: preparation, characterization, and photocatalytic properties. Applied Catalysis B: Environmental, 2012, 115-116: 90-99.

[20] PARIDA K M, REDDY K H, MARTHA S, et al. Fabrication of nanocrystalline $\mathrm{LaFeO}_{3}$ : an efficient Sol-Gel auto-combustion assisted visible light responsive photocatalyst for water decomposition. International Journal of Hydrogen Energy, 2010, 35(22): 12161-12168.

[21] JIA L S, LI J J, FANG W P, et al. Visible-light-induced photocatalyst based on $\mathrm{C}$-doped $\mathrm{LaCoO}_{3}$ synthesized by novel microorganism chelate method. Catalysis Communications, 2009, 10(8): $1230-1234$.

[22] GRINBERG I, WEST D V, TORRES M, et al. Perovskite oxides for visible-light-absorbing ferroelectric and photovoltaic materials. Nature, 2013, 503(7477): 509-512.

[23] WOJCIECH B, EWA A, ANDRZEJ M. The influence of selected parameters on the photocatalytic degradation of azo-dyes in the presence of $\mathrm{TiO}_{2}$ aqueous suspension. Chemical Engineering Journal, 2008, 145(2): 242-248. 